\title{
Type Uncertainty in Ontologically-Grounded Qualitative Probabilistic Matching
}

\author{
David Poole ${ }^{1}$ and Clinton Smyth ${ }^{2}$ \\ 1 Department of Computer Science, University of British Columbia \\ 2 GeoReference Online Ltd., http: / / www.georeferenceonline.com
}

\begin{abstract}
This paper is part of a project to match real-world descriptions of instances of objects to models of objects. We use a rich ontology to describe instances and models at multiple levels of detail and multiple levels of abstraction. The models are described using qualitative probabilities. This paper is about the problem of type uncertainty; what if we have a qualitative distribution over the types. For example allowing a model to specify that a meeting is always scheduled in a building, usually in a civic building, and never a shopping mall can help an agent find a meeting even if it is unsure about the address.
\end{abstract}

\section{Introduction}

In a recent paper [Smyth and Poole, 2004], we described a system for matching instances and models of real-world phenomena. These instances and models have been described by different people using controlled vocabularies (using an ontology) which allow descriptions of model and instances at varied levels of abstraction (using more general or less general terms) and detail (describing objects in terms of parts and subparts or not).

In one practical domain, geological surveys of various countries or provinces publish descriptions of mineral occurrences ${ }^{3}$ (e.g., twelve thousand in British Columbia) at widely varying levels of abstraction and detail. Other people spend careers developing models of archetypical mineral occurrences that can help determine where certain minerals can be found in sufficient quantities to be mined. These models are often at different levels of abstraction and detail from the occurrence descriptions. The problem that we consider is to determine which mineral occurrences fit which models, so that explorers can focus their exploration. This is a case where humans have to make decisions, but they are overwhelmed by the combinatorics. The aim of the computer system is to find the best fitting models to a mineral occurrence or to find the best fitting mineral occurrences to a model. As it is humans who are making the decisions, it is more important to have good explanation facilities and to return multiple potential matches than to return the "best" match according to the computer. Computers help by narrowing down the search space and explaining and justifying the potential matches.

\footnotetext{
${ }^{3}$ In nature there exists a continuum between mineral occurrences and mineral deposits. Geologists tend to call small accumulations of minerals "mineral occurrences", and large ones "deposits". It may take many years to determine whether a mineral occurrence is large enough to be thought of as a deposit.
} 
The main problem is the integration of (qualitative) probabilistic reasoning and the rich ontologies that are needed in such domains. For this paper we'll use the OWL [McGuinness and van Harmelen, 2004; Patel-Schneider, Hayes and Horrocks, 2004] notation where appropriate.

In previous work [Smyth and Poole, 2004], we make the assumption that different descriptions refer to different objects. This assumption is relaxed in this paper. In particular, we allow for uncertainty in the types and allow for qualitative distributions over hierarchically structured classes. By type we mean membership in a class, where the classes are organised hierarchically and are specified as part of an ontology.

This work is quite different to other work on combining probability and ontologies (e.g., P-Classic [Koller, Levy and Pfeffer, 1997]) because we are using the ontologies to construct a rich hypothesis space rather than only having probabilities over the ontologies.

\section{Qualitative Probabilistic Matching}

The general problem is, given a model and a instance, to determine a qualitative value for $P$ (model|instance) that can be used by a human to make decisions. This section gives an overview of our previous paper [Smyth and Poole, 2004].

We decided to use qualitative order-of-magnitude probabilities based on the kappa calculus [Spohn, 1988; Pearl, 1989]. The kappa calculus can be described in terms of surprise; the kappa values correspond to the level of surprise. When probabilities multiply, the corresponding kappa values add, and summing in probability corresponds to minimization in the kappa calculus.

The kappa calculus can be seen as a crude approximation to probability [Darwiche and Goldszmidt, 1994]. This is useful when the probabilities are not available (and may be different for different users) as it gives a rough answer and leaves only a few of the possible matches for a human to evaluate; the implausible matches can be ignored by the human. Note that we use the kappa calculus for the first-level of approximation; we use some finer distinctions to distinguish matches that may have the same values in the kappa calculus. These are described when used.

The instances will have property values that are marked as "present" or "absent".

After feedback from domain experts, we describe the models using a 5 value scale 4 :

- always $p$ : you are very surprised if $p$ is false ${ }^{5}$.

- usually $p$ : you are somewhat surprised if $p$ is false.

- sometimes $p$ : you aren't surprised if $p$ is true or if it's false

- rarely $p$ : you are somewhat surprised if $p$ is true.

- never $p$ : you are very surprised if $p$ is true.

\footnotetext{
${ }^{4}$ None of the theory or results in this paper depends on using this scale, but we will use it in all of our examples. In practice, we have found that experts are happy using this scale, and find it very natural.

5 This is not the always of modal logic. Our experts described things as "always" true even though there were exceptions.
} 
These provide a language for inputting our measures of uncertainty. We output a numerical value in the range $[0,100]$ where 100 is the score for the best possible match and 0 is the score for the worst match. Internally we use a reasonably arbitrary numerical scale that we will use in this paper.

If not for different levels of abstraction and different levels of detail, to compute the qualitative counterpart of $P$ (model|instance), we add the surprises of the instance with respect to the qualitative probabilities specified in the model. The main contribution of [Smyth and Poole, 2004] was in showing how the kappa calculus could be combined with rich ontologies that let us describe models and instances at various levels of abstraction and detail.

As we are adding surprises, and returning the topmost (i.e., least surprising) match, the zero point is arbitrary. We can define the zero to be the level of the empty match ${ }^{6}$ (i.e., a match with an empty description), then we have positive rewards when there is a better match than this and negative rewards (penalties) for those matches that are worse than this.

For each model qualitative probability and for each instance value "present" or "absent", we will have a numerical reward or penalty. Thus, for example, we will talk about the always-present reward (which gives the reward received when a model property that has qualitative probability "always" matches an corresponding instance property that is present) or a usually-absent penalty (when the model property is "usually" present, but it is absent in the instance). For example, if a model specifies a room that is always a bedroom and usually pink and we have an instance that is a bedroom that is not pink (i.e., bedroom is present and pink is absent), we get both the always-present reward and the usually-absent penalty.

Given an abstraction hierarchy of classes, it is important to distinguish the description of an instance from the instance itself. For example, if something is described as a building, it must be some sort of building (generic buildings don't exist). One of the differences between an instance and a model is, when given a general concept, such as "building", in an instance we don't know what sort of building it is, but if the same term is used in a model, we don't care what sort of building it is [Smyth and Poole, 2004]. When we want the probability of an instance, we don't want the probability of the description. The probability of a more abstract description is more likely than that of a more specific instance. For or example, a house is a kind of building, so for any evidence $e, P($ building $\mid e)>P($ house $\mid e)$. However if the model specifies a house, and instance 1 is described as a building and instance 2 is described as a house, then instance 2 definitely fits the model, but it is less likely that instance 1 fits the model.

Smyth and Poole [2004] made two assumptions that we relax in this paper:

- The type of objects was known. That is, there was no qualitative distribution over which class an object is a member of. It did not allow, for example, the model to specify that a place that can take the role of a home office is always a room, usually a bedroom and rarely a master bedroom.

\footnotetext{
6 This is for the case of the open-world assumption, where we don't assume that a complete description is given. We do allow someone to specify that "silence implies absence"; that a part or property that is not described is false. In this case an empty description does not have value zero. It is positive as we expect nothing else and found nothing else.
} 
- In a single description, different descriptions of parts (or other property values) pertain to different parts (or property values). Consider the following two contrasting examples:

- Suppose we had a a model of "a room that Sam likes" that says the room is "usually red and never pink". This could mean that it has a single colour that is usually a non-pink shade of red. Alternatively, there could be multiple colours; as long as one is red and one is non-pink they would be happy. So a blue and pink would be good; they just don't want all-pink or no shade of red. In the first case, the description is referring to a single colour and in the second to multiple colours. Here it seems intuitive that this description is about the same colour.

- Suppose we have a model for a house that always contains a bathroom and always contains a room that is not a bathroom. In this case, we don't want this to refer to the same room (there is no room that is both a bathroom and is not a bathroom), but rather to two rooms.

We need a way to specify we are referring to a single colour or room or to multiple colours or rooms.

Note that these assumptions are interdependent; if we relax the second assumption, we could relax the first by treating class membership as a functional property. However, it is easier to motivate the problems in terms of types, but then treat type as a (functional) property in the algorithm.

\section{Type Uncertainty}

We assume that we have a hierarchy of classes and a hierarchy of properties.

Figure 1 shows a hierarchy where, some of the relations that are true include:

$$
\begin{aligned}
& \langle\text { House, subClass Of, ResidentialBuilding }\rangle \\
& \langle\text { ResidentialBuilding, subClass Of, Building }\rangle \\
& \langle\text { Building, subClass Of, Engineered Artifact }\rangle
\end{aligned}
$$

For this discussion, we do not intend that these are immediate subclasses. There may be many intermediate classes (e.g., that are classes that are subclasses of "Engineered Artifact" and superclasses of "Building"). In this figure, Thing is the topmost class.

Suppose a model that specifies that the location of some activity is:

always a Building and rarely a House.

Call this model $M_{1}$.

Given a simple description of an instance where we only give a single class that is present, let's determine how surprised we are that the location is at that instance. The description could be any position in the taxonomy, and the instance could be any leaf that is a descendent of the class that is said to be present. The figure shows the five qualitatively different regions of the taxonomy that the description of an instance could be in:

- Region 1. If the description is in region 1, the cousins of Building (the values in the same tree that are neither descendents nor ancestors of Building), the instance is not a Building. 


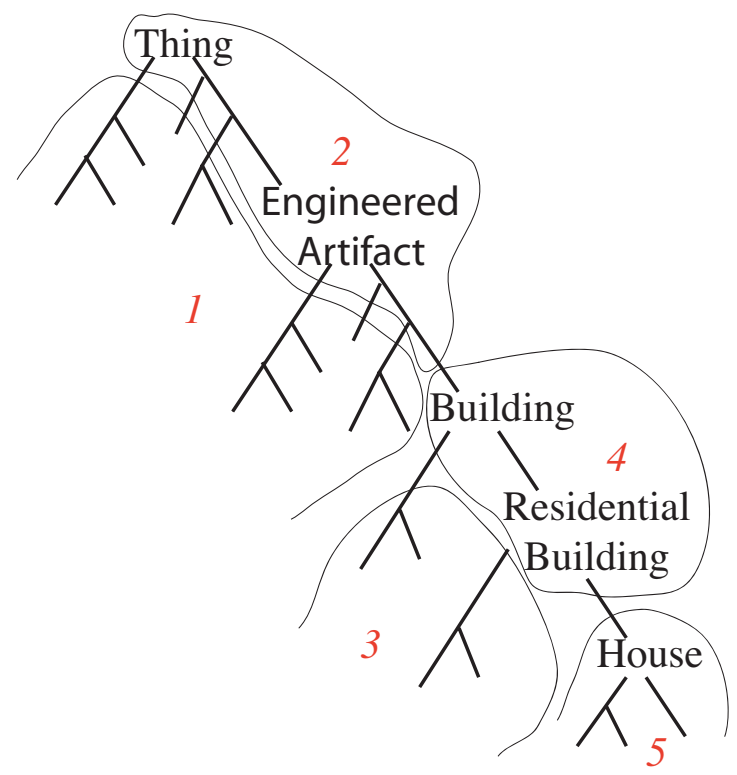

Fig. 1. A class hierarchy. Different exceptional regions for model $M_{1}$ are numbered.

- Region 2. The description is an ancestor of Building so the instance is perhaps a Building, but could be a non-building.

- Region 3. The instance is a Building and not a House.

- Region 4. The instance is a Building and perhaps a House.

- Region 5. The instance is a House.

Consider how surprised we would be that an object in each of these locations would be an instance of the model "always a Building and rarely a House".

If the instance description is in region 1, the instance would receive the alwaysabsent penalty. The model says the instance is always a building and the instance is not a building.

Suppose description $d_{2}$ of the instance is in region 2, for example it is the description Engineered Artifact. We don't know if the instance is a Building or not. To understand the qualitative probability, consider the probability of the model $M_{1}$ given the description $d_{2}$ :

$$
\begin{aligned}
P\left(M_{1} \mid d_{2}\right)= & P\left(M_{1} \mid \text { Building } \wedge d_{2}\right) * P\left(\text { Building } \mid d_{2}\right) \\
& +P\left(M_{1} \mid \neg \text { Building } \wedge d_{2}\right) * P\left(\neg \text { Building } \mid d_{2}\right)
\end{aligned}
$$

$P\left(M_{1} \mid\right.$ Building $\left.\wedge d_{2}\right)=P\left(M_{1} \mid\right.$ Building $)$ as the model doesn't specify anything more general that Building. Similarly, $P\left(M_{1} \mid \neg\right.$ Building $\left.\wedge d_{2}\right)=P\left(M_{1} \mid \neg\right.$ Building $)$. Thus

$$
\begin{aligned}
P\left(M_{1} \mid d_{2}\right)= & P\left(M_{1} \mid \text { Building }\right) * P\left(\text { Building } \mid d_{2}\right) \\
& +P\left(M_{1} \mid \neg \text { Building }\right) * P\left(\neg \text { Building } \mid d_{2}\right)
\end{aligned}
$$


In terms of the kappa-calculus (taking logs, ignoring adding by zero, and minimizing):

$$
\kappa\left(M_{1} \mid d_{2}\right)=\min \left(\kappa\left(\text { Building } \mid d_{2}\right), \kappa\left(M_{1} \mid \neg \text { Building }\right)\right.
$$

assuming that

$-\kappa\left(\neg\right.$ Building $\left.\mid d_{2}\right)=0$; we are not surprised that the Engineered Artifact is not a building. We would be surprised if it is a building as there are many more sorts of engineered artifacts than buildings.

$-\kappa\left(M_{1} \mid\right.$ Building $)=0$; we are not surprised that a building matches the model $M_{1}$ as the model $M_{1}$ specifies the object is always a building.

Thus the "surprise" that the engineered artifact fits the model comes from either the surprise that the Engineered Artifact is a building or the surprise that a non-building matches the model. Our level of surprise is the minimum of these two.

We could have surprise information as part of our ontology. For each element in the taxonomy, we would have a value of how surprising each child is. For example, we could infer the surprise of Building given the description Engineered Artifact.

If we didn't have the information in the taxonomy, we can make some simplifying assumptions to estimate this value. Suppose the description $d_{2}$ is $m$ levels in the hierarchy above Building, and suppose that the average branching factor of $b$, and that the children of any node have approximately equal probability. Then $P\left(\right.$ Building $\left.\mid d_{2}\right) \approx(1 / b)^{m}$. Taking logarithms, we see that the surprise that the instance is a Building should be linear in $m$.

We do not use the kappa calculus directly, as this would entail having a surprise that there is no description. We'd rather just ignore non-existent descriptions. This can be done by defining the surprise value of a empty description as zero. We get positive rewards for being less surprised than this and negative rewards (penalties) for being more surprised. Given that we know something exists, not specifying a value is the same as stating it has the top value (Thing in the above taxonomy). This then gives us a way to calibrate the surprise due to the reasons above. The value is zero when the description is Thing. If the description is not Thing, but in region 2, then is should have a positive reward, as it is more likely a Building than if it were a Thing. Under the assumptions made above, this should be linear in the depth. The main assumption is that surprises are not given in the taxonomy and that all children are approximately equal ${ }^{7}$.

If the assumption that children are approximately equal is not a reasonable assumption, it is possible to specify the surprise values for each child in the taxonomy as part of the ontology. For example, specifying how surprised you are that residential building is a house. Note that in this case it is possible to specify a model that is surprised by a normal condition; in this case, the model should also be surprised by a empty description. For example, if things are usually engineered artifacts, but a model specifies that fits to the model are rarely engineered artifacts, then the model should be surprised by a description of an object just as Thing.

\footnotetext{
${ }^{7}$ Note that this means all children along the path from Thing are approximately equal, not that a child is equal to all of its sibling
} 
If the instance is in region 5, it gets the rarely-present penalty. We know the instance is a type of house but the model specifies that we should be surprised by the meeting in a house.

In order to understand the reward of an instance in region 3, it is instructive to consider some more models. Suppose model $M_{0}$ is "always a Building" and $M_{2}$ is "always a Shopping Centre". Then we have $M_{2}$ subsumes $M_{1}$ (given that Shopping Centre is a subClass Of of Building and is disjoint with House) and $M_{1}$ subsumes $M_{0}$. If the instance is a Strip Mall (a subClass of Shopping Centre), then this instance matches all three models. As $M_{0}$ and $M_{2}$ give the same match; an always-present reward, it seems reasonable to also give the match with $M_{1}$ the same reward and to not also give it a rarely-absent reward.

Instances in region 4, are known to be buildings and they could be houses. If we do a similar probabilistic analysis to region 2, with $d_{4}$ a description on region 4 , we get:

$$
\begin{aligned}
P\left(\text { model } \mid d_{4}\right)= & P(\text { model } \mid \text { House } \wedge \text { Building }) * P\left(\text { House } \mid d_{4}\right) \\
& +P(\text { model } \mid \neg \text { House } \wedge \text { Building }) * P\left(\neg \text { House } \mid d_{4}\right)
\end{aligned}
$$

(as $P\left(\right.$ Building $\left.\mid d_{4}\right)=1$ ). If you just consider the second part of the sum, you are not surprised that the model is true for a building that is not a house (it is "always" true), you are also not surprised that a building in region 4 is not a house. Thus in terms of the kappa values, this has kappa value 0 . That is, kappa $\left(\right.$ model $\left.\mid d_{4}\right)=0$.

However, this is considered to be a worse match than for an instance in region 3 , and so gets a small penalty that is proportional to the depth of the description. This value is designed to be dominated by the kappa values so that it only distinguished instances that have the same kappa values.

\section{Matching Algorithm}

For this paper ${ }^{8}$, we assume that we are given an ontology that specifies:

- A class hierarchy

- Domains and ranges for all properties

- Declarations that properties are functional

For this paper we make a number of simplifying assumptions (that are not made in out full system):

- There are no property hierarchies. Property hierarchies complicate the matching.

- The class hierarchies are trees. Siblings in the class hierarchies form disjoint sets of individuals.

- The open world assumption: we do not assume that we are told everything about an individual. If we want to say that something is not true, we need to say that its value is absent.

\footnotetext{
${ }^{8}$ Our ontology specifies other information such as property hierarchies, and number ranges, that are outside of the scope of this paper.
} 
- Properties are functional. Note that this also applies to the "type" property as an instance only has a single type (even if we don't know what it is).

We assume that we are given an instance or instances of the form:

$$
\langle\text { Object, Property, Value, Frequency }
$$

where Frequency is either present or absent and reference specifies the source of the information.

We assume that we are given a model or models of the form:

$$
\langle\text { Object, Property, Value, Frequency〉 }
$$

where Frequency is one of \{always, usually, sometimes, rarely, never\}.

In the models and instances, the Values can be primitive values (such as strings or numbers or pair of numbers representing ranges), descriptions of other objects, or references to other objects.

For example, Figure 2 gives a model of a research meeting. As part of the ontology, we assume that the range of starts_at is a time. Thus we know that the type of $t_{1}$ must be a subclass of time. That is why it isn't specified here. Similarly for the location $L_{1}$.

\begin{tabular}{|l|l|l|l|}
\hline Object & Property & Value & Frequency \\
\hline$M_{1}$ & has_location & $L_{1}$ & usually \\
$M_{1}$ & starts_at & $T_{1}$ & always \\
$M_{1}$ & organized_by & $P_{1}$ & always \\
$L_{1}$ & type & Building & always \\
$L_{1}$ & type & House & rarely \\
$T_{1}$ & between & {$[900,1700]$} & always \\
$T_{1}$ & between & {$[1200,1259]$} & rarely \\
$P_{1}$ & type & Admin_staff & usually \\
$P_{1}$ & type & Dept_head & rarely \\
$P_{1}$ & type & Financial_officer & never \\
\hline
\end{tabular}

Fig. 2. An example model of a research meeting

Note that the first line of this description allows for the fact that a meeting may not have a location (which is different from whether we know its location).

Under these assumptions, there is a canonical form for instances. Because the declarations are implicitly conjoined, you can assume there is exactly one "present" class for any functional property (including type) and a number of absent classes that are subclasses of the present class. This is because you can always assume that the top element is present, and if a class and a subclass are both present, you can remove the superclass as present and preserve the meaning. There can't be two classes that are both "present" if one is not a subclass of the other if the hierarchies are trees (as there are no elements in common between the classes). 
Similarly we can assume that for a model, there is at most one always, at most one usually, that frequencies go down in the hierarchy, there are no children of never or cousins of always. The only cousins of a usually are nevers. [Note that sometimes is used when we have a complete knowledge assumption; it will be ignored in this section.]

Figure 3 gives an algorithm to determine the score for an instance matching a model. We use the notation I.present to be the position in the hierarchy of the value of the instance that is declared to be present. Similarly M.always is the position in the hierarchy of the value declared to be always true in model M. Conditions involving M.always are assumed to be false if nothing is declared to be always true in model $M$. Below and above refer to positions in the hierarchy (above is more general), and a node is below itself and is above itself.

One non-obvious aspect of this algorithm is when the model has an "always" above a "usually". In this case, if an instance has present below the the always, but a cousin of the usually, it gets just the usually-absent penalty, and no reward for the always-present. This is reasonable as, if the always was not there, this would be equivalent to having always at the top. If the instance has present above the usually, it has a reward that is linear in the depth of the present instance, independent of the position of the always.

\section{Matching Parts}

In order to complete the algorithm, we need to consider the case where the value may be an object that has a complex description. In this case, when the model and the instance both have have a complex descriptions, we need to determine the match between these complex descriptions. If the instance has multiple instances of the property (and the property is not functional), we choose the best match (independently of the model frequency).

For example, consider the value of the organized_by property of $M_{1}$ in Figure 2 . If there exists a person who fits the description of $P_{1}$, there should be a reward, and if there doesn't there should be a penalty.

Again it is constructive to consider a full probabilistic analysis of the probability of model $M_{1}$ given instance $I_{1}$ :

$$
\begin{aligned}
P\left(M_{1} \mid I_{1}\right)= & P\left(M_{1} \mid\left(\exists P_{1}\right) \wedge I_{1}\right) P\left(\left(\exists P_{1}\right) \mid I_{1}\right) \\
& +P\left(M_{1} \mid\left(\neg \exists P_{1}\right) \wedge I_{1}\right) P\left(\left(\neg \exists P_{1}\right) \mid I_{1}\right) \\
= & P\left(M_{1} \mid\left(\exists P_{1}\right)\right) P\left(\left(\exists P_{1}\right) \mid I_{1}\right) \\
& +P\left(M_{1} \mid\left(\neg \exists P_{1}\right)\right) P\left(\left(\neg \exists P_{1}\right) \mid I_{1}\right)
\end{aligned}
$$

where $\exists P_{1}$ is shorthand for there exists a $P_{1}$ that matches the description of $P_{1}$ in Figure 2 . The model gives the qualitative values $P\left(M_{1} \mid\left(\exists P_{1}\right)\right)$. To compute the qualitative values of $P\left(\left(\exists P_{1}\right) \mid I_{1}\right)$ we can find the best match of $P_{1}$ to the values of organized_by in $I_{1}$.

Taking the qualitative version of this formula, we replace multiplication by addition and the addition by minimum in the kappa calculus or maximum in our system. The works if we expect $\left(\exists P_{1}\right)$ to be true (the frequency of organized_by in $M_{1}$ is always or usually) and we find that we have positive support for $\left(\exists P_{1}\right)$. 


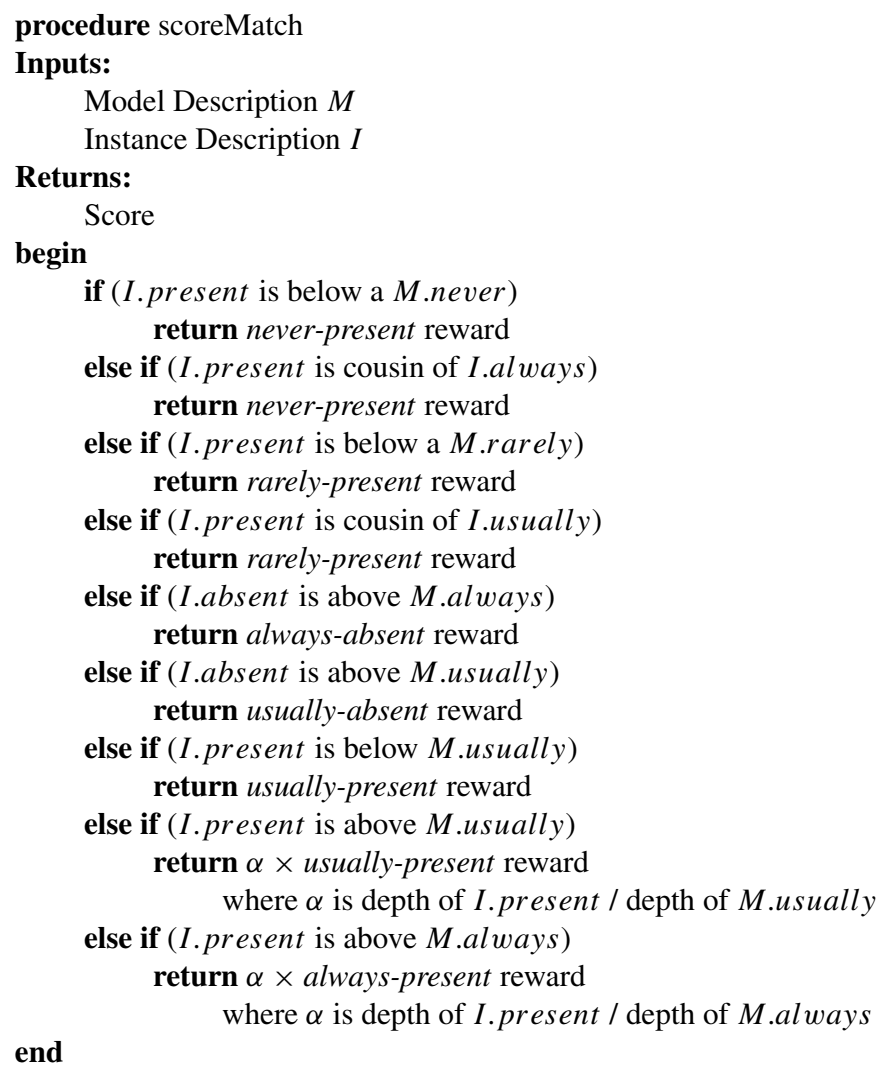

Fig. 3. Determining Reward from Instance and Model Descriptions

Unfortunately, the other 3 cases are not as straightforward as we cannot readily compute kappa $\left(\left(\neg \exists P_{1}\right) \mid I_{1}\right)$ as if $\left(\exists P_{1}\right)$ has no surprise, then its negation has some surprise, but we don't know how much, and $\left(\exists P_{1}\right)$ has some surprise, then its negation has no surprise. We have chosen a simple scheme that gives intuitive results, as follows.

If the model specifies we are surprised that $\left(\exists P_{1}\right)$ (the frequency is "rarely") and we are not surprised that it is true in the instance (i.e., $\left(\exists P_{1}\right) \mid I_{1}$ is positive), we get the surprise of the rarely (the rarely-present reward).

If the qualitative probability of $\left(\exists P_{1}\right)$ kin $I_{1}$ is negative, we give the appropriate always-absent, usually-absent, ...never-absent reward.

For example, if the instance had multiple organizers, we need to determine which one best fulfills the role specified in the model. In terms of the kappa calculus, the distribution over which instance fulfills the role becomes a minimization of surprised (maximization of scores). We do this by choosing the one with the highest score. Then we consider the model frequency and how surprised we are than an organizer of the appropriate type exists. 


\section{Conclusion}

This paper has grown out of a project to build knowledge-based decision tools in various domains such as minerals exploration, geological hazards (landslides, earthquakes), land-use planning. We have needed to have qualitative reasoning and rich ontologies. We don't have the probabilistic knowledge or the utilities to do full decision theory, but have developed a system that uses a small but natural set of qualitative probabilities that can integrate with the ontologies being developed and with the sort of knowledge about instances and models that can be obtained. This paper outlined how we are handling cases where a functional relation has qualitative probabilistic constraints on what values it can take (some are more surprising than others). We have made some pragmatic choices that seem to work in practice, but there is much more theoretical and empirical work that needs to be carried out.

\section{References}

Darwiche, A. and Goldszmidt, M. [1994]. On the relation between kappa calculus and probabilistic reasoning, UAI-94, pp. 145-153.

Koller, D., Levy, A. and Pfeffer, A. [1997]. P-classic: A tractable probabilistic description logic, AAAI-97, Providence, RI, pp. 390-397.

McGuinness, D. L. and van Harmelen, F. [2004]. Owl web ontology language overview, W3C Recommendation 10 February 2004, W3C.

URL: http://www.w3.org/TR/owl-features/

Patel-Schneider, P. F., Hayes, P. and Horrocks, I. [2004]. Owl web ontology language: Semantics and abstract syntax, W3C Recommendation 10 February 2004, W3C.

URL: http://www.w3.org/TR/owl-semantics/

Pearl, J. [1989]. Probabilistic semantics for nonmonotonic reasoning: A survey, in R. J. Brachman, H. J. Levesque and R. Reiter (eds), KR-89, Toronto, pp. 505-516.

Smyth, C. and Poole, D. [2004]. Qualitative probabilistic matching with hierarchical descriptions, Proc. Ninth International Conference on the Principles of Knowledge Representation and Reasoning (KR-04).

URL: http://www.cs.ubc.ca/spider/poole/papers/KR04SmythC.pdf

Spohn, W. [1988]. A general non-probabilistic theory of inductive reasoning, UAI-88, pp. 315-322. 